\title{
Low-Temperature Fabrication of Titanium Metal/Barium Titanate Composite Capacitors Containing Core-Shell Particles and Their Dielectric Properties
}

\author{
Shintaro Ueno ${ }^{1 *}$, Yasunao Sakamoto ${ }^{1}$, Kouich Nakashima ${ }^{1}$, and Satoshi Wada ${ }^{1}$ \\ 1 Interdisciplinary Graduate School of Medicine and Engineering, University of Yamanashi, \\ 4-4-37 Takeda, Kofu 400-8510, Japan \\ * Corresponding author: e-mail: sueno@yamanashi.ac.jp
}

\begin{abstract}
To develop the high-volume energy storage device based on ceramic capacitors, we attempted that Ti metal particles working as internal electrodes were distributed in barium titanate (BT) layers. We compared two kinds of the metal particles; uncoated Ti metal particles and Ti-BT core-shell particles ( $\mathrm{Ti}$ metal particles with thin BT coating layers). The green compacts consisting of the metal particles, titanium oxide precursor particles, and barium titanate fillers were successfully converted into the Ti/BT composite compacts by the hydrothermal method without heating procedure. The effective dielectric constant of these composites tends to increase with a metal content, and then drastically increases up to over $10^{3}$ near the percolation threshold (insulator-metal transition point). These dielectric constant behaviors can be explained by the percolation theory. By using the core-shell particles, the percolation threshold increases from 0.609 to 0.734 , suggesting that the BT shell layers were effective to suppress the current leakage.

Key words: composite capacitor, dielectric properties, percolation materials, hydrothermal method
\end{abstract}

\section{INTRODUCTION}

It has been attempted to develop the high-volume energy storage devices based on ceramic capacitors by fabricating conductor/dielectrics composites [1,2]. In these composites, the conductors act as internal electrodes and the capacitance of composites is significantly enhanced. Generally, an effective dielectric constant of conductor/dielectrics composite capacitors categorized as percolation materials increases with a content of conductive particles distributed in the dielectric layers, and then drastically increases by several orders of magnitude close to a percolation threshold $\left(f_{C}\right.$ : an insulator to metal transition point). Such a unique dielectric constant behavior is frequently interpreted by a percolation theory [3] and is represented as following equation $[2,4]$,

$$
<_{\varepsilon}>=\varepsilon_{m}\left[\left(f_{C}-f\right) / f_{C}\right]^{-q}
$$

where $<_{\varepsilon}>$ is the effective dielectric constant, $\varepsilon_{m}$ is the dielectric constant of matrix, $f_{C}$ is the percolation threshold, $f$ is the filling factor, and $q$ is the critical exponent. Actually, many research groups reported the fabrication of metal/dielectric composites with the high effective dielectric constant. Pecharromán, et al. reported the $\mathrm{Ni} /$ barium titanate $\left(\mathrm{BaTiO}_{3}, \mathrm{BT}\right)$ composite materials with a high, frequency-independent effective dielectric constant $\left(<_{\varepsilon}>\approx 80000\right)$, fabricated by sintering the mixture of $\mathrm{Ni}$ particles and BT particles at higher temperature in an $\mathrm{Ar} / \mathrm{H}_{2}$ gas [1]. The high effective dielectric constant of 500000 was also reported for the $\mathrm{Mo} /$ mullite composites prepared by sintering in vacuum [2]. Since these metal/dielectric ceramics composites were usually fabricated by a conventional solid-phase synthesis including high-temperature sintering procedures, the kinds of available metal are significantly limited and, in addition, these composites need to be sintered under reduction atmosphere to avoid oxidization of metals except for noble metals. On the other hand, some research groups proposed lower-temperature fabrication processes of metal/dielectric ceramics composites by a hydrothermal electrophoresis deposition method [5-7]. In this method, the metal layers play a role in electrodes and react to dielectrics. Thus, the metals only with two dimensional structures such as plates and foils have been demonstrated and the relatively small dielectric constant was reported for these composite capacitors due to the geometric limitation [7].

In this paper, we propose that low-temperature fabrication process of metal/dielectric ceramics composites by using a hydrothermal method; green compacts consisting of Ti metal particles and $\mathrm{TiO}_{2}$ particles (precursor particles of dielectric layers) are converted into $\mathrm{Ti} / \mathrm{BT}$ composite compacts by the hydrothermal treatment in $\mathrm{Ba}(\mathrm{OH})_{2}$ solution at a temperature below $200{ }^{\circ} \mathrm{C}$. Additionally, to suppress the current leakage of the metal/dielectric composites, we employed the Ti (core)-BT (shell) particles alternative to the pure Ti metal particles and compared the dielectric properties of the resultant Ti/BT composites.

\section{EXPERIMENTAL}

2.1 Fabrication of Ti-BT core-shell particles

Two kinds of metal particles were employed in this experiment. Ti metal powders were purchased by the company (particle size $<45 \mu \mathrm{m}$, Wako Pure Chemical) and the Ti-BT core-shell particles were prepared from the Ti metal powders by the hydrothermal method [8]. $1.0 \mathrm{~g}$ of the Ti metal powders were put into a Teflon container filled with $7.5 \mathrm{~mL}$ of $\mathrm{Ba}(\mathrm{OH})_{2} \cdot 8 \mathrm{H}_{2} \mathrm{O}$ (Wako Pure Chemical, 99.9\%) aqueous solution (50 $\left.\mathrm{mmol} \cdot \mathrm{dm}^{-3}\right)$. Then, the hydrothermal treatment was 
carried out at $230{ }^{\circ} \mathrm{C}$ for $12 \mathrm{~h}$ in an autoclave. The resultant particles were washed with water and methanol, and dried at $80^{\circ} \mathrm{C}$

\subsection{Fabrication of Ti/BT composite compacts}

The Ti metal particles or the Ti-BT core-shell particles were mixed with rutile-type $\mathrm{TiO}_{2}$ nanoparticles (MPT-851, Ishihara Sangyo Kaisha), BT nanoparticles (BT05; particle size is approximately $500 \mathrm{~nm}$, Sakai Chemical Industry), and acetone by an ultrasonic stirrer (USS-1, Nihonseiki Kaisha Ltd., Japan) until acetone was evaporated. The molar ratio of $\mathrm{TiO}_{2} / \mathrm{BT}$ was fixed at 1.0 , and the mixing ratios of the metal particles in the precursor powders were varied. The resultant mixed powders were pressed into green compacts by a uniaxial press at $625 \mathrm{MPa}$. Each green compact was placed in a Teflon container filled with $7.5 \mathrm{~mL}$ of $\mathrm{Ba}(\mathrm{OH})_{2} \cdot 8 \mathrm{H}_{2} \mathrm{O}$ aqueous solution $\left(50 \mathrm{mmol} \cdot \mathrm{dm}^{-3}\right)$ and the hydrothermal treatment was carried out at $160{ }^{\circ} \mathrm{C}$ for $24 \mathrm{~h}$ in an autoclave. The obtained compacts were washed with water and methanol, and dried at $80{ }^{\circ} \mathrm{C}$.

\subsection{Characterization}

The relative density and the metal content of the compacts were measured by an Archimedes method. Crystal structures of the samples were identified by an X-ray diffraction (XRD, Ultima IV, Rigaku, CuKa, 40 $\mathrm{kV}, 30 \mathrm{~mA}$ ). For the XRD measurements, the powder samples were prepared by grinding the composite compacts. Microstructures of the samples were observed by a scanning electron microscope (SEM, JSM-6510, JEOL). For electrical measurements, gold electrodes were sputtered on the top and bottom surfaces of samples and these samples were cut down to a size of $2 \times 2 \mathrm{~mm}^{2}$. The dielectric properties were measured at frequency ranged from $100 \mathrm{~Hz}$ to $100 \mathrm{MHz}$ at room temperature by an impedance analyzer (HP4294A, Agilent). Before the electrical measurements, the samples were dried at $200{ }^{\circ} \mathrm{C}$ for $1 \mathrm{~h}$ in vacuum to remove the adsorbed water.

\section{RESULTS AND DISCUSSION}

3.1 Microstructures of Ti/BT composite compacts

To confirm the formation of the core-shell particles, microstructures of the $\mathrm{Ti}$ metal and the core-shell particles were observed by the SEM (Fig.1). While the smooth surface can be found for the Ti metal particles (Fig.1(a)), the nanoparticle layer formed on the surface of the micron-size $\mathrm{Ti}$ metal particles after the hydrothermal treatment (Fig.1(b)). According to the XRD measurement, the core-shell particles consisted of $\mathrm{Ti}$ metal and BT. In the previous report, the BT nanoparticle layer formed on the surface of the Ti metal

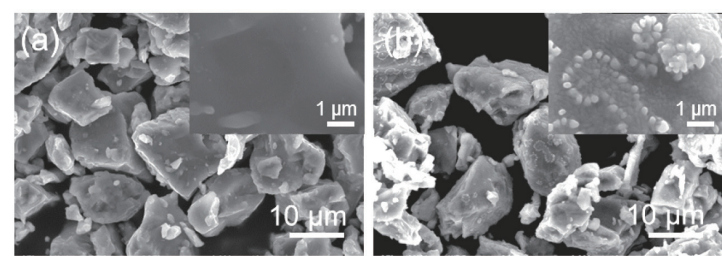

Fig.1 SEM images of (a) the Ti metal and (b) the Ti-BT core-shell particles used in this experiment (The insets indicate the surface of particles at high magnification).
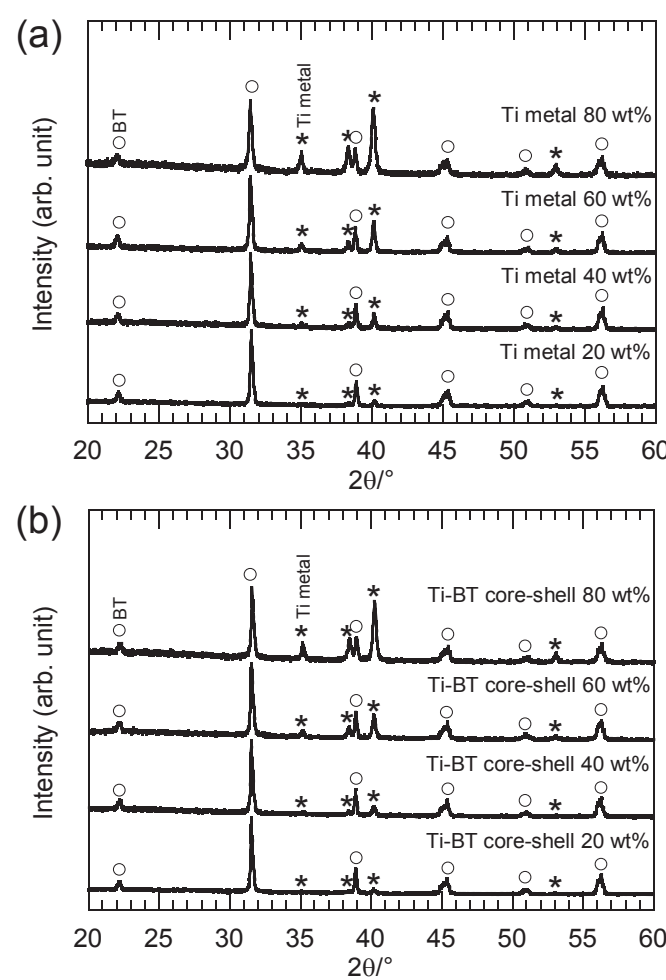

Fig.2 XRD patterns of the ground powders of the composites containing various amounts of (a) the $\mathrm{Ti}$ metal particles and (b) the Ti-BT core-shell particles.

particles by a chemical reaction with $\mathrm{Ba}(\mathrm{OH})_{2}$ in hydrothermal conditions [8]. It is considered that the BT nanoparticle layers were constructed by a local dissolution of the surface of Ti metal and deposition of BT nanoparticles [8,9]. The thickness of the BT nanoparticle layers is estimated for about $10-100 \mathrm{~nm}$ by a mass increase of the Ti metal powders after the hydrothermal treatment. This estimation is based on the assumption that $\mathrm{Ti}$ spherical particles were covered by homogeneous BT thin layers.

The size and shape of the composite compacts did not change, but all the compacts became more rigid after the hydrothermal treatment. XRD patterns of ground powders of the composites after the hydrothermal treatment are shown in Fig. 2. All the diffraction peaks are indexed by $\mathrm{Ti}$ metal and $\mathrm{BT}$, and thus $\mathrm{TiO}_{2}$ is considered to be completely converted into BT by the following chemical reactions [10].

$$
\begin{aligned}
& \mathrm{TiO}_{2}+2 \mathrm{H}_{2} \mathrm{O} \rightarrow \mathrm{Ti}(\mathrm{OH})_{4} \mathrm{aq} \\
& \mathrm{Ti}(\mathrm{OH})_{4} \mathrm{aq}+\mathrm{Ba}(\mathrm{OH})_{2} \rightarrow \mathrm{BaTiO}_{3}+3 \mathrm{H}_{2} \mathrm{O}
\end{aligned}
$$

For all the samples, yields of the deposited BT on the compacts are estimated to be more than $80 \%$ based on mass increases of the compacts. The relative density and the Ti metal content of the Ti/BT composite compacts are summarized in Tables 1 and Table 2. The metal content shown in the tables means a volume fraction of the metal component to a total volume of the compact including a pore volume. After the hydrothermal treatment, the relative density of all the Ti/BT composite compacts increases because the chemical reaction from $\mathrm{TiO}_{2}$ to $\mathrm{BT}$ is accompanied by a volume increase. 
Table 1 A Relative density of the composites before and after the hydrothermal treatment and a metal content of the Ti/BT composites containing Ti metal particles with various mixing ratios.

\begin{tabular}{cccc}
\hline Metal & \multicolumn{2}{c}{ Relative density (\%) } & Metal \\
\cline { 2 - 3 } mixing ratio & before & after & content (\%) \\
\hline $20 \mathrm{wt} \%$ & 68.7 & 81.0 & 15.6 \\
$40 \mathrm{wt} \%$ & 73.8 & 83.5 & 31.9 \\
$60 \mathrm{wt} \%$ & 81.1 & 88.5 & 51.0 \\
$80 \mathrm{wt} \%$ & 85.5 & 90.9 & 70.0 \\
\hline
\end{tabular}

Table 2 A Relative density of the composites before and after the hydrothermal treatment and a metal content of the Ti/BT composites containing Ti-BT core-shell particles with various mixing ratios.

\begin{tabular}{cccc}
\hline Metal & \multicolumn{2}{c}{ Relative density (\%) } & Metal \\
\cline { 2 - 3 } mixing ratio & before & after & content (\%) \\
\hline $20 \mathrm{wt} \%$ & 68.5 & 78.2 & 15.4 \\
$40 \mathrm{wt} \%$ & 73.2 & 84.6 & 32.5 \\
$60 \mathrm{wt} \%$ & 79.5 & 87.1 & 50.3 \\
$80 \mathrm{wt} \%$ & 84.0 & 89.1 & 69.1 \\
\hline
\end{tabular}

Additionally, the relative density of the Ti/BT composites monotonically increases with a metal mixing ratio. This is because metal particles can transform to fill the space by the uniaxial press. Actually, the same tendency can be seen in the green compacts before the hydrothermal treatment. The relative density and the metal contents are comparable at the same metal mixing ratio for these $\mathrm{Ti} / \mathrm{BT}$ composites.

SEM images of fractured cross sections of the Ti/BT composites are shown in Fig.3. The nano- and submicron-sized particles aggregated in the compacts are considered as the BT particles because the Ti metal particles used in this experiment were micron-size. A wide size-distribution of the BT particles suggests that both nucleation and crystal growth of BT proceeded during the hydrothermal treatment. In Fig.3, a part of these BT particles formed three-dimensionally connected networks, and thereby the compacts became more rigid. This technique is applicable to fabrication of composite compacts including low-thermostability materials. In Fig.3 (c) and (d), the flat surface of the metal particles can be seen (pointed by arrows), where the Ti metal surfaces were exposed even after the hydrothermal treatment. In these regions, electric paths are easy to be formed, resulting in the percolation at the lower metal content. On the other hand, the flat Ti metal surface could not be observed for the composites containing the core-shell particles. Hence, it is important to form the BT shell layers on Ti metal particles before preparing the green compacts.

\subsection{Dielectric properties of Ti/BT composite compacts}

The variations of the impedance and the phase of the Ti/BT composites with a metal content are shown in Fig.4. Regardless of the presence or absence of BT shell layers on the Ti metal particles, the impedance gradually decreases with a metal content, and then drastically decreases due to the insulator to metal transition; the metal content of $60.9 \mathrm{vol} \%$ and $73.4 \mathrm{vol} \%$ for the composites containing the uncoated $\mathrm{Ti}$ metal and the core-shell particles, respectively. The abrupt change in

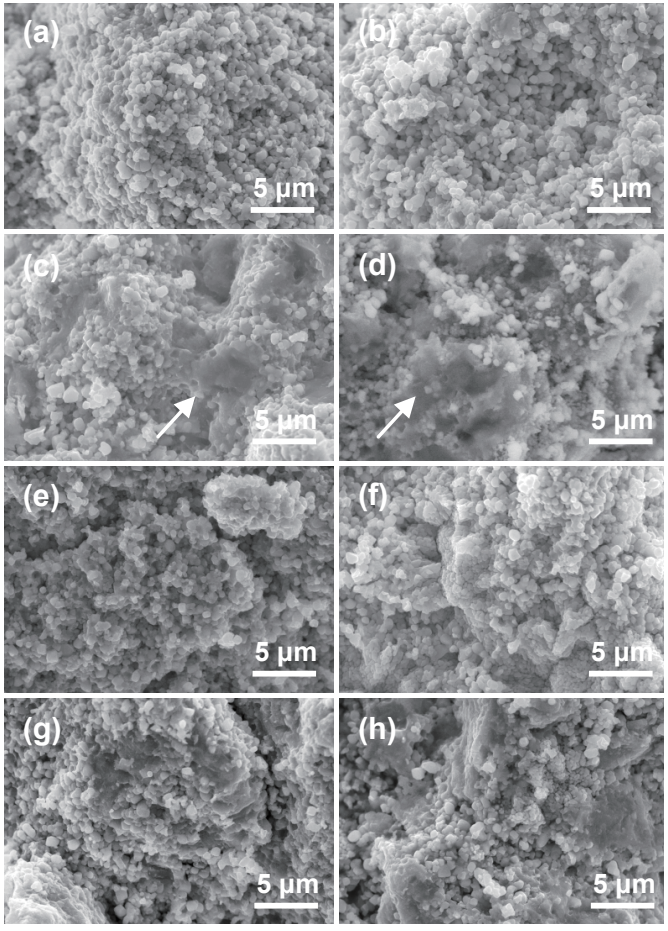

Fig.3 SEM images of the fractured surface of the Ti/BT composites with the various mixing ratios of the $\mathrm{Ti}$ metal particles ((a) 20, (b) 40, (c) 60, and (d) $80 \mathrm{wt} \%$ ) and the Ti-BT core-shell particles ((e) 20, (f) 40, (g) 60, and (h) $80 \mathrm{wt} \%)$.

the phase at the transition points also suggests the current leakage. Accordingly, we determined that the percolation threshold, $f_{C}$, is 0.609 and 0.734 for the $\mathrm{Ti} / \mathrm{BT}$ composites containing the $\mathrm{Ti}$ metal and the core-shell particles, respectively. The higher $f_{C}$ value of the Ti/BT composites containing the core-shell particles suggests that the BT shell layers on the Ti metal particles spatially prevented the direct contacts among the Ti metal particles and suppressed the current leakage [11].

The effective dielectric constant and the loss tangent of these composites were measured at room temperature, and the variation of these values with a metal content in the composites is shown in Fig.5. For both of the Ti/BT composites, the effective dielectric constant tends to increase with a metal content, and the drastic increase is found near the $f_{C}$. Meanwhile, the loss tangent is almost constant at a lower metal content and slightly increases near the $f_{C}$. These effective dielectric constant behaviors are similar to the percolation materials previously reported in literatures [1,2]. Simulation curves of the effective dielectric constant based on the percolation theory (Eq. 1) using our experimental data are depicted by dashed lines in Fig.5 (only the data calculated at a frequency of $100 \mathrm{~Hz}$ are shown). The values used for the calculations are as follows; $\varepsilon_{m}=716, q=0.96, f_{C}=0.609$ for the composites containing the uncoated $\mathrm{Ti}$ metal particles, and $\varepsilon_{m}=687, q=0.88$, and $f_{C}=0.734$ for the composites containing the Ti-BT core-shell particles. The dashed lines are roughly consistent with the dielectric behavior of the Ti/BT composites. Thus, we consider that the relatively high effective dielectric constant at a higher metal content is attributed to the 

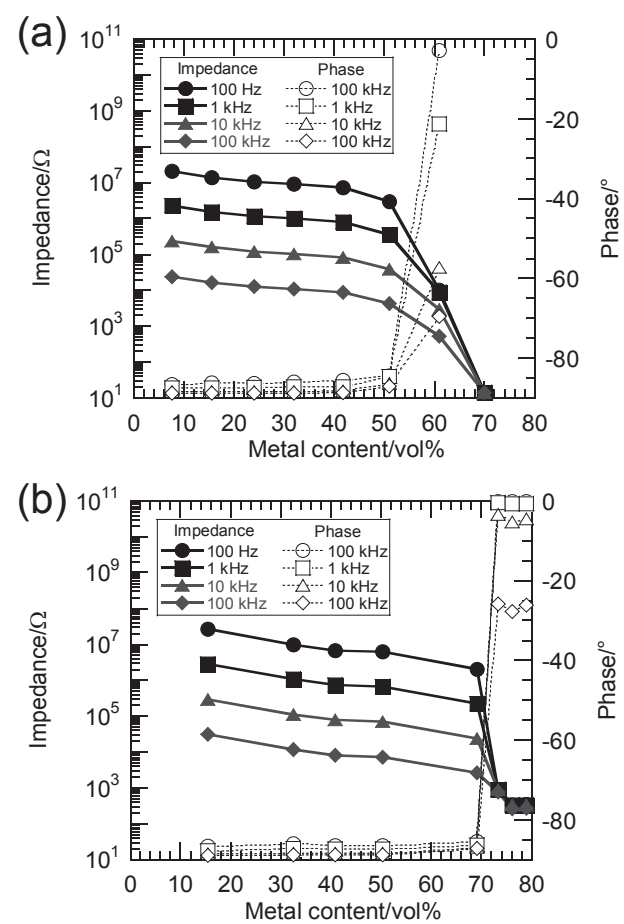

Fig.4 The variation of the impedance and the phase with a metal content for the Ti/BT composites containing (a) Ti metal particles and (b) Ti-BT core-shell particles.

percolation effect.

In this study, the Ti/BT composite capacitors could be fabricated by the wet chemical approach without heating procedure exhibited the relatively high effective dielectric constant over $10^{3}$. According to the percolation theory, the conductivity of the Ti/BT composites also increases with a metal content. We tried to measure the dielectric breakdown strength $\left(E_{b}\right)$ of these composites. The measured $E_{b}$ was still much lower level $\left(E_{b}=29 \mathrm{kV} / \mathrm{cm}\right.$ and $E_{b}=7 \mathrm{kV} / \mathrm{cm}$ for the composite containing $24.0 \mathrm{vol} \%$ of the Ti metal and $50.3 \mathrm{vol} \%$ of the core-shell particles, respectively) and for the samples containing the higher volume fraction of metal components, the $E_{b}$ was unmeasurable because of their significantly low resistivity. At an applied electric field well below the $E_{b}$, the current density was order of $10^{-7}$ $\mathrm{A} / \mathrm{mm}^{2}$, and therefore, the enhancement in the $E_{b}$ is needed for the energy-storage applications. For this purpose, the distributed metal particles should be sufficiently separated by high-quality dielectric layers, if possible, without grain boundaries and pores. Accordingly, we are trying to elaborate the microstructures of the metal/dielectrics composites by controlling crystal growth in the hydrothermal process to form highly crystalline dielectric layers.

\section{CONCLUSION}

The Ti/BT composite compacts containing the uncoated $\mathrm{Ti}$ metal particles or the Ti-BT core-shell particles with various metal contents were fabricated by the hydrothermal treatment at $160{ }^{\circ} \mathrm{C}$ without heating procedures. The effective dielectric constant of these composites tends to increase with a metal content up to over $10^{3}$ near the percolation threshold. These unique dielectric constant behaviors can be explained by the
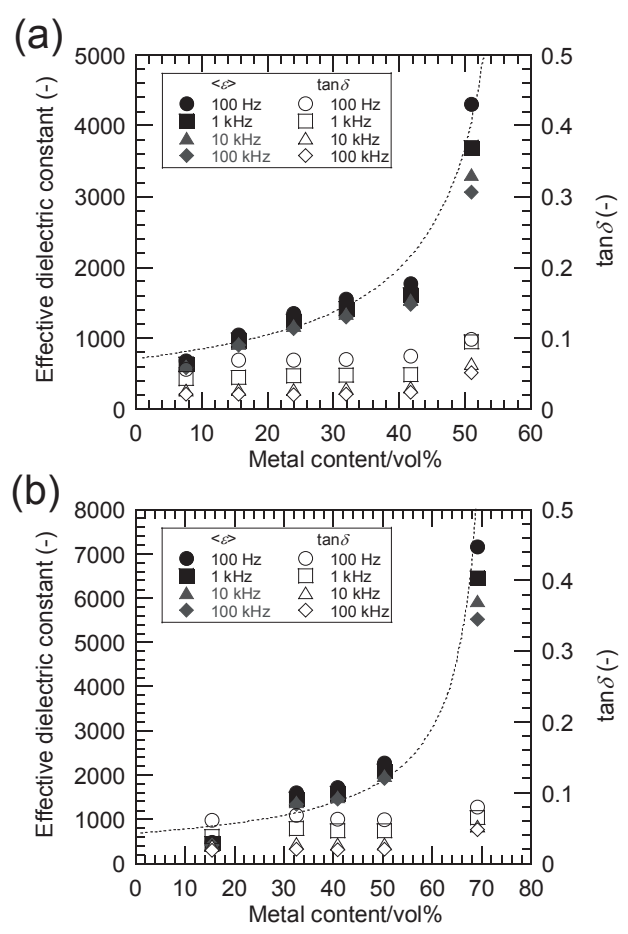

Fig.5 The variation of the effective dielectric constant and the loss tangent with a metal content for the Ti/BT composites containing (a) $\mathrm{Ti}$ metal particles and (b) Ti-BT core-shell particles. The dashed lines indicate the variation of effective dielectric constant at a frequency of $100 \mathrm{~Hz}$ assumed by the percolation theory.

percolation theory. The BT shell layers on the Ti metal particles prevented the direct contact among Ti metal particles and suppressed the current leakage. It resulted in an increase in the percolation threshold. The dielectric properties of such metal/dielectric ceramics composites depend largely on their microstructures.

\section{REFERENCES}

[1] C. Pecharromán, F. Esteban-Betegón, J. F. Bartolomé, S. López-Esteban, and J. S. Moya, $A d v$. Mater., 13, 1541-1544 (2001)

[2] C. Pecharromán and J. S. Moya, Adv. Mater., 12, 294-297 (2000)

[3] A. L. Efros and B. I. Shklovskii, Phys. Stat. Sol. B, 76, 475-485 (1976)

[4] J. S. Moya, S. Lopez-Esteban, and C. Pecharromán, Prog. Mater. Sci., 52, 1017-1090 (2007)

[5] R. Bacsa, P. Ravindranathan, and J. P. Dougherty, J. Mater. Res., 7, 423-428 (1992)

[6] W.-D. Yang and K.-M. Hung, J. Mater. Sci., 37, 1337-1342 (2002)

[7] W. Zhu, S. A. Akbar, R. Asiaie, and P. K. Dutta, J. Electroceram., 2, 21-31 (1998)

[8] R. R. Basca and J. P. Dougherty, J. Mater. Sci. Lett., 14, 600-602 (1995)

[9] P. Bendale, S. Venigalla, J. R. Ambrose, E. D. Verink, Jr., and J. H. Adair, J. Am. Ceram. Soc., 76, 2619-2627 (1993)

[10] P. K. Dutta, R. Asiaie, S. A. Akbar, and W. Zhu, Chem. Mater., 6, 1542-1548 (1994)

[11] H.-Y. Chang, S.-Y. Cheng, C.-I. Sheu, and Y.-H. Wang, Nanotechnol., 14, 603-608 (2003)

(Received February 2, 2014; Accepted April 7, 2014) 